\title{
Aspherical manifolds, relative hyperbolicity, simplicial volume and assembly maps
}

\author{
IGOR BELEGRADEK
}

This paper contains examples of closed aspherical manifolds obtained as a by-product of recent work by the author [3] on the relative strict hyperbolization of polyhedra. The following is proved.

(I) Any closed aspherical triangulated $n$-manifold $M^{n}$ with hyperbolic fundamental group is a retract of a closed aspherical triangulated $(n+1)$-manifold $N^{n+1}$ with hyperbolic fundamental group.

(II) If $B_{1}, \ldots B_{m}$ are closed aspherical triangulated $n$-manifolds, then there is a closed aspherical triangulated manifold $N$ of dimension $n+1$ such that $N$ has nonzero simplicial volume, $N$ retracts to each $B_{k}$, and $\pi_{1}(N)$ is hyperbolic relative to $\pi_{1}\left(B_{k}\right)$ 's.

(III) Any finite aspherical simplicial complex is a retract of a closed aspherical triangulated manifold with positive simplicial volume and non-elementary relatively hyperbolic fundamental group.

20F65

\section{Introduction}

Recently there has been a surge of interest in relatively hyperbolic groups (see Drutu and Sapir [17], Osin [34], Dahmani [8], Groves [21] and Yaman [42]). Three basic classes of examples are finite free products (which are hyperbolic relative to the factors), hyperbolic groups (which are hyperbolic relative to the trivial subgroups), and geometrically finite isometry groups of negatively pinched Hadamard manifolds (which are hyperbolic relative to the maximal parabolic subgroups). Finally, any group is hyperbolic relative to itself; we call a relatively hyperbolic group non-elementary unless it is finite, or virtually- $\mathbb{Z}$, or hyperbolic relative to itself.

A major source of examples of relatively hyperbolic groups is the small cancellation theory (see Osin [35]) which typically involves a 2-dimensional construction, such as adding a "sufficiently long" relation to the group. In [3] the author showed that the spaces obtained via relative strict hyperbolization of polyhedra have relatively 
hyperbolic fundamental groups, which allows to construct many higher-dimensional relatively hyperbolic groups. Here is the main result of this note.

Theorem 1.1 If $B^{n}$ is a closed aspherical triangulated $n$-manifold such that $\pi_{1}\left(B^{n}\right)$ is hyperbolic relative to the subgroups $H_{1}, \ldots, H_{k}$, then there is a closed aspherical triangulated $(n+1)$-manifold $N^{n+1}$ and an embedding $i: B^{n} \rightarrow N^{n+1}$ such that

- $N^{n+1}$ retracts onto $i\left(B^{n}\right)$,

- $N^{n+1}$ has nonzero simplicial volume, and

- $\pi_{1}\left(N^{n+1}\right)$ is hyperbolic relative to $i_{*}\left(H_{1}\right), \ldots, i_{*}\left(H_{k}\right)$.

Theorem 1.1 is also true in PL and smooth categories, meaning that the embedding $i: B^{n} \rightarrow N^{n+1}$ and the retraction $N^{n+1} \rightarrow i\left(B^{n}\right)$ are either PL or smooth. I do not know whether Theorem 1.1 holds in the topological category. If each $H_{i}$ is trivial, then Theorem 1.1 reduces to (I), while (II) follows from Theorem 3.1, which is a slight generalization of Theorem 1.1. The item (III) follows from (II) combined with the reflection group trick of Davis [11] implying that each finite aspherical simplicial complex $K$ is a retract of a closed aspherical manifold $M$.

The reflection group trick was used with great success by Davis and collaborators to produce closed aspherical manifolds with various exotic properties (see Davis [13] and references therein), eg if $\pi_{1}(K)$ is not residually finite, then neither is $\pi_{1}(M)$, because residual finiteness is inherited by subgroups. Similarly, Theorem 1.1 gives a new source of closed aspherical manifolds with nonzero simplicial volume and various exotic properties. Adapting an example of Weinberger, we build in each dimension $\geq 5$ a closed aspherical manifold $N$ with nonzero simplicial volume such that $\pi_{1}(N)$ is a non-elementary relatively hyperbolic group with unsolvable word problem. In particular, $\pi_{1}(N)$ is not hyperbolic, not $C A T(0)$, not automatic, not residually finite, and not linear over any commutative ring.

Also (I) yields numerous closed aspherical triangulated manifolds with hyperbolic fundamental group, which admit no obvious locally $C A T(-1)-$ metric. In fact, the hyperbolicity of the fundamental group follows by an indirect argument that uses Dahmani's combination theorem for relatively hyperbolic groups [9], and Osin's result that if all the maximal parabolic subgroups of a relatively hyperbolic group have linear Dehn function, then the ambient relatively hyperbolic group is hyperbolic [34].

More generally, if we specify a class of groups $\mathcal{C}$, eg abelian, nilpotent, slender etc, then given a closed aspherical triangulated manifold $B$ with $\pi_{1}(B)$ hyperbolic relative to subgroups that belong to $\mathcal{C}$, Theorem 1.1 produces manifolds with the same properties in each dimension $>\operatorname{dim}(B)$. 
Just like in [13, Section 11] we have applications to the assembly maps, eg (I) implies that if the Assembly Map Conjecture (that the assembly maps in $L$-theory and $K$ theory are isomorphisms) holds for all closed aspherical triangulated $(n+1)$-manifolds with hyperbolic fundamental groups, then it also holds for all closed aspherical triangulated $n$-manifolds with hyperbolic fundamental groups. Similarly, (II) shows that the Assembly Map Conjecture for closed aspherical triangulated manifolds is equivalent to the Assembly Map Conjecture for closed aspherical triangulated manifolds with nonzero simplicial volume and non-elementary relatively hyperbolic fundamental group. Finally, (III) implies that if the Novikov Conjecture holds for all non-elementary relatively hyperbolic fundamental groups which are fundamental groups of closed triangulated aspherical manifold with nonzero simplicial volume, then it holds for the fundamental groups of all finite aspherical complexes. All these results on assembly maps also hold for PL or smooth manifolds.

\section{Relative strict hyperbolization}

We refer to Gromov [19], and to the work of Charney, Davis, Januszkiewicz and Weinberger $[6 ; 15 ; 14]$ for general background on hyperbolization of polyhedra, and to [3] for a detailed study of relative strict hyperbolization.

For the purposes of this paper the relative strict hyperbolization is a procedure that takes as an input a compact connected triangulated $n$-manifold pair $(M, \partial M)$ and produces another triangulated $n$-manifold pair $(R, \partial R)$ together with a surjective continuous map $h:(R, \partial R) \rightarrow(M, \partial M)$ that restricts to a homeomorphism of the boundaries $\partial R \rightarrow \partial M$, induces surjections on homology and the fundamental group, and pulls back rational Pontrjagin classes and the first Stiefel-Whitney class. All this also works in smooth category by working with smooth triangulations.

Also $\partial R$ is incompressible in $R$ (ie no homotopically nontrivial loop in $\partial R$ is nullhomotopic in $R$ ), and if each path-component of $\partial M$ is aspherical, then $R$ is aspherical. Furthermore, the space obtained from $R$ by attaching a cone on $\partial R$ is a locally $C A T(-1)$ piecewise-hyperbolic simplicial complex, and according to [3] $\pi_{1}(R)$ is non-elementary relatively hyperbolic, relative to the fundamental groups of the pathcomponents of $\partial R$ that are infinite.

\section{Main result}

All the proofs in the section hold without change in either for triangulated, PL, or smooth manifolds; for brevity we just deal with the PL case. 
Theorem 3.1 If $B_{1}^{n}, \ldots B_{m}^{n}$ are closed aspherical PL $n$-manifolds with $n>0$ such that for each $k=1, \ldots m$, the group $\pi_{1}\left(B_{k}^{n}\right)$ is hyperbolic relative to the subgroups $H_{1}^{k}, \ldots, H_{m_{k}}^{k}$, then there is a closed aspherical PL $(n+1)$-manifold $N^{n+1}$ and PL embeddings $i_{k}: B_{k}^{n} \rightarrow N^{n+1}$ such that the following hold.

- For each $k=1, \ldots m$, there is a retraction $r_{k}: N^{n+1} \rightarrow i_{k}\left(B_{k}^{n}\right)$.

- $\pi_{1}\left(N^{n+1}\right)$ is hyperbolic relative to $\left\{i_{k *}\left(H_{j}^{k}\right): k=1, \ldots m, j=1, \ldots m_{k}\right\}$.

- $N^{n+1}$ has nonzero simplicial volume.

Proof Let $M$ be the connected sum of all the manifolds $B_{k} \times[0,1]$; since $n>0$, the manifold $M$ is connected. For any fixed $k$, we can collapse to the point each summand $B_{j} \times[0,1]$ with $j \neq k$, which defines a retraction $M \rightarrow B_{k} \times[0,1]$. Therefore, $M$ retracts onto each $B_{k}$. Now apply the relative strict hyperbolization to $(M, \partial M)$ to obtain $(R, \partial R)$, and look at the double $D R$ of $R$ along $\partial R$. The double $N^{n+1}:=D R$ retracts onto each $B_{k}$ as follows. First apply the quotient map of the double involution, then use $h:(R, \partial R) \rightarrow(M, \partial M)$, then use the retraction $M \rightarrow B_{k}$ constructed above, and finally go back to $\partial R$ via $h^{-1}$.

By [3] $\pi_{1}(R)$ is hyperbolic relative to $\pi_{1}\left(B_{k}\right)$ 's, so a combination theorem for relatively hyperbolic groups due to Dahmani [9] implies that $\pi_{1}(D R)$ is hyperbolic relative to $\pi_{1}\left(B_{k}\right)$ 's. Hence, by a recent result of Drutu-Sapir [17, Corollary 1.14] $\pi_{1}(D R)$ is hyperbolic relative to the images of $H_{j}^{k}$ under $\pi_{1}$-injective inclusions $B_{k} \rightarrow D R$.

To see that the double $D R$ has nonzero simplicial volume, note that the simplicial volume is nonincreasing under continuous maps, and the quotient map $D R \rightarrow D R / R=$ $R / \partial R$ maps the fundamental class $[D R]$ onto the the fundamental class of the pseudomanifold $R / \partial R$, which is locally $C A T(-1)$ [3, Remark 3.2]; in fact, $R / \partial R$ is the strict hyperbolization of the space obtained from $M$ by attaching a cone over $\partial M$. Hence $R / \partial R$ has positive simplicial volume (see Yamaguchi [41]). (Instead of using [41], one could employ a more general result of Mineyev [30] that since $R / \partial R$ is aspherical and $\pi_{1}(R / \partial R)$ is hyperbolic, every cohomology class in $H^{n+1}(R / \partial R, \mathbb{R})$ is bounded for $n>0$, which implies by a standard argument (see Benedetti and Petronio [4, Proposition F.2.2]) that $\|R / \partial R\|>0$ ).

Proof of (I) of the abstract We keep the notations of Theorem 3.1 with $m=1$. By assumption $\pi_{1}\left(B_{1}\right)$ is hyperbolic, and hence, by a theorem of Osin [34, Corollary 2.41] we can remove $\pi_{1}\left(B_{1}\right)$ 's from the list of maximal parabolic subgroups of $\pi_{1}(D R)$, so that $\pi_{1}(D R)$ is hyperbolic. Alternatively, instead of referring to the Osin's result one could a result of Drutu-Sapir [17, Corollary 1.14] to conclude that $\pi_{1}(D R)$ is hyperbolic relative to the trivial subgroup, and hence is hyperbolic. 
Proof of (II) of the abstract Since any group is hyperbolic relative to itself, we can let $m_{k}=1$ and $H_{1}^{k}:=\pi_{1}\left(B_{k}\right)$ for each $k=1, \ldots, m$, so Theorem 3.1 yields the desired assertion.

Remark 3.2 One can show that $D R$ is orientable if and only if each $B_{k}$ is orientable. Indeed, if $D R$ is orientable, then so is each $B_{k}$ because it sits in $D R$ with trivial normal bundle. Conversely, if each $B_{k}$ is orientable, then so is $M$, and hence $R$ is orientable because the relative strict hyperbolization preserves orientability. Now $D R$ is orientable as the double of an orientable manifold.

Remark 3.3 If $m=1$, one can improve the previous remark to the claim that the retraction $r_{1}$ pulls back the first Stiefel-Whitney class $w_{1}$. In fact, $r_{1}$ is the composition of three retractions, and each of them pulls back $w_{1}$. This is obvious for the projection $M=B_{1} \times[0,1] \rightarrow B_{1}$ that in fact pulls back the stable tangent bundle (and this is what fails if $m>1$ ). Also the hyperbolization map $R \rightarrow M$ pulls back $w_{1}$ essentially because the hyperbolic manifold with corners used as the building block in the strict hyperbolization is orientable, see [3, Remark 3.2]. Finally, an elementary covering space argument shows that the retraction $D R \rightarrow R$ always pulls back $w_{1}$.

\section{Closed aspherical manifolds with nonzero simplicial vol- ume}

There are very few known ways to produce closed aspherical manifolds of nonzero simplicial volume. Denote by $\mathcal{P}$ the class of closed manifolds of nonzero simplicial volume, and by $\mathcal{A P} \subset \mathcal{P}$ the subclass of manifolds in $\mathcal{P}$ that are aspherical.

The classes $\mathcal{P}$ and $\mathcal{A P}$ are closed under the following operations:

(1) products (see Gromov [18, page 10]).

(2) finite covers, quotients by free actions of finite groups, and homotopy equivalences to a closed manifold [18, page 8].

(3) taking surface bundles (ie if $V$ is in $\mathcal{P}$, and $\Sigma$ is an orientable closed surface of $\chi(\Sigma)<0$, then the total space of any smooth orientable $\Sigma$-bundle over $V$ is in $\mathcal{P}$, and by the homotopy exact sequence of the fibration, if the same holds when $\mathcal{P}$ is replaced with $\mathcal{A} \mathcal{P}$ ) (see Hoster and Kotschick [24]).

Remark 4.1 Manifolds in $\mathcal{P}$ can be also produced by gluing compact manifolds with positive relative simplicial volume along incompressible boundary components that 
have amenable fundamental groups (see Gromov [18, page 55], and Kuessner [26]. Furthermore, the resulting manifold is in $\mathcal{A P}$ if all the pieces are aspherical, and all the boundary components are aspherical and incompressible (because any graph of spaces with aspherical edges and vertices is aspherical provided the edge-to-vertex maps are $\pi_{1}$-injective).

The class $\mathcal{A P}$ contains the following manifolds:

- closed negatively curved Riemannian manifolds (this is due to Thurston, who also proved positivity of relative simplicial volume for open complete negatively pinched manifolds of finite volume, see [18, Section 1.2],

- closed aspherical manifolds with hyperbolic fundamental groups (see Mineyev [30]) for example, the manifolds obtained by strict hyperbolization (see Charney and Davis [6]),

- closed locally symmetric manifolds of nonpositive curvature (see Lafont and Schmidt [27]),

- doubles of finite volume negatively pinched manifolds. More generally, one can glue several manifolds as in Remark 4.1, provided at least one piece has nonzero relative simplicial volume, as happens eg for closed aspherical Haken 3 -manifolds with at least one hyperbolic piece in the JSJ-decomposition (see Soma [39]).

The above lists all sources of manifolds $\mathcal{A P}$ known to the author before writing this note. There are some other manifolds in $\mathcal{P}$, eg the manifolds that appear as the base of flat affine bundle with nonzero Euler number (see Gromov [18, page 23], Smillie [38] and Hausmann [23]). Unfortunately, I do not know whether any of these examples lie in $\mathcal{A P}$, with the obvious exception of closed orientable surfaces of negative Euler characteristic, or their products, which are already included in the above lists.

In this section we add more items to the above list by exploring Remark 4.1. We start from the following observation.

Proposition 4.2 If $M$ is a compact orientable PL $n$-manifold, with $n \geq 2$, such that the pseudomanifold $M / \partial M$ admits a locally-CAT(-1) metric. Then any closed orientable manifold $N$ containing $M$ as a codimension zero PL submanifold has nonzero simplicial volume.

Proof If $N$ is an arbitrary closed orientable manifold $N$ containing $M$ as a codimension zero PL-submanifold, then the quotient $\bar{Z}$ of $N$, obtained by collapsing 
$N \backslash \operatorname{Int}(M)$ to the point, is homeomorphic to $M / \partial M$, which is an oriented pseudomanifold whose fundamental class is the image of the fundamental class of $N$ under the quotient map $N \rightarrow \bar{Z}$. Since simplicial volume is nonincreasing under continuous maps, we deduce $\|N\| \geq\|\bar{Z}\|$. The cohomology class dual to the fundamental class of $Z$, is bounded by Mineyev [30] as $n \geq 2$. Hence $\|\bar{Z}\|>0$ by a standard argument (see, for example, Benedetti and Petronio [4, Proposition F.2.2]).

Example 4.3 If $M$ is any manifold of dimension $\geq 2$ obtained obtained by relative strict hyperbolization, then $M / \partial M$ admits a piecewise-hyperbolic locally-CAT(-1) metric as was noted in [3]. Note that $M$ is aspherical if and only if each component of $\partial M$ is aspherical; in this case the double of $M$ is also aspherical so by Proposition 4.2 it lies in $\mathcal{A P}$, and more generally we could glue $M$ with several other aspherical manifolds and the result will be in $\mathcal{A P}$ provided all their boundary components are aspherical and incompressible.

Example 4.4 If $M$ is a compact manifold with boundary obtained by chopping off cusps of a complete finite volume real hyperbolic manifold, and if each component of $\partial M$ is a flat torus of injectivity radius $>\pi$, then $M / \partial M$ admits a locally $-C A T(-1)$ metric (see Mosher and Sageev [31]). Again the double of $M$ lies in $\mathcal{A P}$. Furthermore, there are other ways to embed $M$ into a closed aspherical manifold, eg by cusp closing (see Schroeder [37]), or by Dehn surgery on $\partial M$ (see [1], where Anderson constructs Einstein metrics on these manifolds). Here the ambient closed manifolds are aspherical, because they can be shown to admit metrics of nonpositive sectional curvature via Gromov-Thurston $2 \pi$-Theorem.

Remark 4.5 The assumption of Example 4.4 that the injectivity radii of flat components of $\partial M$ are $>\pi$ cannot be dropped, eg any hyperbolic knot complement in the 3-sphere, or the punctured torus sitting inside the 2-torus are codimension zero submanifolds of the closed manifolds whose simplicial volume vanishes.

Remark 4.6 Since the fundamental groups of finite volume complete hyperbolic manifolds are residually finite, any such manifold has a finite cover satisfying the assumptions of Example 4.4.

Remark 4.7 Gromov stated in [20, page 189] that a result similar to Example 4.4 should be true in all negatively curved symmetric spaces. I do not know how to prove this claim. 


\section{Simplicial volume vs nonpositive curvature}

One may naively suspect that all manifolds in $\mathcal{A P}$ admit metrics of nonpositive sectional curvature. It seems this problem was first discussed by Hoster and Kotschick [24], who noted that the surface bundles over surfaces belong to $\mathcal{A P}$ while some of them admit no nonpositively curved metrics, by the work of Kapovich and Leeb [25]. Also there are manifolds in $\mathcal{A P}$ that are homeomorphic but not diffeomorphic to nonpositively curved ones (see Aravinda and Farrell [2], Okun [32], and Ontaneda [33]).

Perhaps, the simplest example can be constructed by chopping off cusps of a finite volume complete locally symmetric manifold of non-constant sectional curvature, and then doubling it along the boundary. Because the simplicial volume is additive under gluings along boundary incompressible components with amenable fundamental groups (see Kuessner [26]) the double has nonzero simplicial volume, yet it admits no locally-CAT(0) metric because its fundamental group contains a nonabelian nilpotent subgroup coming from the cusp (see Bridson and Haefliger [5, page 439]).

This idea can be pushed much farther by noting that by the item (III) in the abstract the fundamental group of any finite aspherical complex embeds into $\pi_{1}(D)$ for some $D$ in $\mathcal{A P}$, so it is very easy to find examples when $\pi_{1}(D)$ is not $C A T(0)$. For example, following the idea of Mess [28], one finds $D$ in $\mathcal{A P}$ such that $\pi_{1}(D)$ contains the Baumslag-Solitar group $B S(1,2)$, which in turn contains a subgroup of dyadic rationals, which cannot happen for $C A T(0)$ groups. Here is a more sophisticated example based on an observation of Weinberger [13, Section 13].

Corollary 5.1 For any $n \geq 5$ there exists a closed aspherical PL $n$-manifold with nonzero simplicial volume, and non-elementary relatively hyperbolic fundamental group that has unsolvable word problem.

Proof By work of Collins and Miller [7] there exists a finite aspherical 2-complex $X_{0}$ whose fundamental group has unsolvable word problem. By a theorem of Stallings, $X_{0}$ is homotopy equivalent to a finite aspherical complex $X$ that embeds into $\mathbb{R}^{n}$ for each $n \geq 4$ (see Stallings [40], and Dranišnikov and Repovš [16]). So the reflection group trick produces a closed aspherical PL $n$-manifold $B$ that retracts onto $X$, and by Theorem 3.1 there exists a closed aspherical PL manifold $D$ of dimension $n+1$ with nonzero simplicial volume, and non-elementary relatively hyperbolic fundamental group that retracts onto $X$. If a group has a solvable word problem, so do all of its finitely generated subgroups, so the word problem of $\pi_{1}(D)$ is unsolvable.

Remark 5.2 Note that the word problem of a finitely presented group $H$ is solvable if $H$ is $C A T(0)$ (see Bridson and Haefliger [5, page 441]) or hyperbolic, or automatic, 
or asynchronously automatic, or residually finite, or linear over any commutative ring (see Miller [29] and references therein). Thus the group in Corollary 5.1 satisfies none of these properties.

\section{Applications to assembly maps}

To motivate the discussion of this section we mention, following Davis [10], some outstanding conjectures on the assembly maps in $L$-theory and $K$-theory

$$
A_{\pi}^{L}: H_{*}(B \pi ; \mathbf{L}) \rightarrow L_{*}(\mathbb{Z} \pi) \quad A_{\pi}^{K}: H_{*}(B \pi ; \mathbf{K}) \rightarrow K_{*}(\mathbb{Z} \pi)
$$

which are still open even when the Eilenberg-MacLane space $B \pi$ can be realized as a closed aspherical manifold.

Novikov Higher Signatures Conjecture For any group $\pi$, the rational assembly maps $A_{\pi}^{L} \otimes \operatorname{id}_{\mathbb{Q}}$ and $A_{\pi}^{K} \otimes \mathrm{id}_{\mathbb{Q}}$ are injective.

Assembly Map Conjecture If $\pi$ is torsion-free, the assembly maps $A_{\pi}^{L}$ and $A_{\pi}^{K}$ are isomorphisms.

It is known that the assembly map in algebraic $K$-theory is always injective in degrees $*<2$ with cokernels $K_{*}(\mathbb{Z} \pi), \widetilde{K}_{0}(\mathbb{Z} \pi), \mathrm{Wh}(\pi)$ for $*<0, *=0, *=1$, respectively. Thus the validity of the Assembly Map Conjecture in $K$-theory yields significant topological information. Other topological consequences can be obtained via the surgery exact sequence, in which the surgery obstruction map is closely related to the $L$-theory assembly map. One of the key conjectures in geometric topology is the following.

Relative Borel Conjecture If $M$ is a compact aspherical manifold with possibly nonempty boundary, then any homotopy equivalence of manifold pairs $(N, \partial N) \rightarrow$ $(M, \partial M)$ that restricts to a homeomorphism $\partial N \rightarrow \partial M$ must be homotopic, relative to the boundary, to a homeomorphism $N \rightarrow M$.

It is known that if $M$ is a compact aspherical manifold of dimension $\geq 5$, then the Relative Borel Conjecture for $M$ holds if and only if $\mathrm{Wh}\left(\pi_{1}(M)\right)$ vanishes and the Assembly Map Conjecture in $L$-theory holds for $\pi_{1}(M)$.

For the purposes of this paper, the key property of the assembly map is naturality (which can be deduced eg from Hambleton and Pedersen [22]). More precisely, the $K$-theory assembly map is natural with respect to any group homomorphism $\phi: G \rightarrow \pi$, while 
the $L$-theory assembly is only natural under the twisting preserving homomorphisms. Namely, the definition of $L(\mathbb{Z} \pi)$ involves a twisting, ie a homomorphism $w(\pi): \pi \rightarrow$ $\mathbb{Z}_{2}$, and we require that $w(G)=w(\pi) \circ \phi$. If $\pi$ is the fundamental group of a manifold $M$, then there is a natural twisting coming from the first Stiefel-Whitney class of $M$.

Suppose we have a retraction of groups $G \rightarrow \pi$, eg the retraction of the fundamental groups induced by the retraction of aspherical spaces in (I), (II). To see the idea of what follows, note that since the Whitehead group depend covariantly on the group, we get an induced retraction $\mathrm{Wh}(G) \rightarrow \mathrm{Wh}(\pi)$, in particular, if $\mathrm{Wh}(G)$ vanishes, then so does $\operatorname{Wh}(\pi)$.

More generally, let $A$ : $\mathbf{Q} \rightarrow \mathbf{P}$ be a natural transformation of two covariant functors $\mathbf{Q}$, $\mathbf{P}$ defined on and taking values in the category of groups and group homomorphisms. Applying $A$ to the inclusion $\pi \rightarrow G$ and the retraction $G \rightarrow \pi$, we get the following commutative diagram.

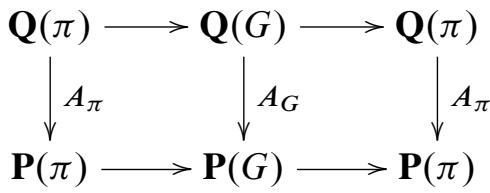

Since the rows are induced by the retraction, the composition of the two horizontal maps in each row is the identity, so the first map is injective, and the second map is onto. An easy diagram chase shows that if $A_{G}$ is injective, then so is $A_{\pi}$, and moreover, the same statement holds if the word "injective" is replaced by "surjective", or "isomorphism", or "split injective". Again, to apply the above to the $L$-theory assembly map we have to assume that the retraction $G \rightarrow \pi$ preserves the twisting, but this assumption is not very restrictive because eg given any twisting $w: \pi \rightarrow \mathbb{Z}_{2}$, there is a twisting of $G$ that makes $G \rightarrow \pi$ twisting-preserving, namely, the twisting obtained by composing $G \rightarrow \pi$ and $w$.

I suspect that this idea has been well-known for a long time, eg since any closed aspherical $n$-manifold $M^{n}$ is a retract of a closed aspherical $(n+1)$-manifold, namely $M^{n} \times S^{1}$, one sees that if the Assembly Map Conjecture holds for all closed aspherical $(n+1)$-manifolds, then it also holds all closed aspherical $n$-manifolds. Here the retraction $M^{n} \times S^{1} \rightarrow M$ preserves the twisting given by the first Stiefel-Whitney class $w_{1}$, because it pulls back the stable tangent bundle.

Davis used this idea in [13, Sections 11] to show that the Assembly Map Conjecture holds for all closed aspherical manifolds, it also holds of all groups with $B \pi$ a finite complex. Indeed, by the reflection group trick any finite aspherical simplicial complex $K$ is a retract of a closed aspherical manifold $M$. Furthermore, given any twisting 
$w: \pi_{1}(K) \rightarrow \mathbb{Z}_{2}$ one can even choose $M$ with $w_{1}(M)=w \circ r_{*}$, where $r: M \rightarrow K$ denotes the retraction. Indeed, $w$ can be realized as the first Stiefel-Whitney class of a compact manifold $F$, that is a thickening of $K$ (eg embed $K$ into a Euclidean space, take a regular neighborhood, and then let $F$ to be the line bundle over the regular neighborhood with the first Stiefel-Whitney class $w$ ). Now use $F$ as the fundamental chamber in the reflection group trick to produce a closed aspherical manifold $M$ that retracts onto $F$, and hence onto $K$. By a result of Davis [12, Proposition 1.4], the retraction $M \rightarrow F$ pulls back the stable tangent bundle, so it preserves the twisting.

Finally, specializing to the setting of Theorem 3.1, we get, among other things, the results on the Assembly Map Conjecture and the Novikov Conjecture stated in the introduction. By Remark 3.3 if $m=1$, then the retraction $r_{1}$ preserves the natural twistings given by $w_{1}$.

\section{Questions}

Question 7.1 Are there closed aspherical manifolds with hyperbolic fundamental group which are not homotopy equivalent to a triangulated manifold? If you drop the requirement that the fundamental group is hyperbolic, such examples were constructed by Davis and Januszkiewicz [14].

Question 7.2 Suppose we start from a finite volume real hyperbolic manifold with toral cusp cross-section, and fill them by a "sufficiently complicated" Dehn surgery (see Anderson [1]) so that the result is a closed aspherical manifold $M$ of nonpositive sectional curvature. Under what conditions $M$ has nonzero simplicial volume? (By Example 4.4 this is true if each cusp has a cross-section of injectivity radius $>\pi$ ). In particular, do the aspherical homology 4-spheres constructed by Ratcliffe and Tschantz [36] have nonzero simplicial volume? Note that there are many homology 3 -spheres carrying hyperbolic metrics, so that their simplicial volumes are nonzero.

\section{Acknowledgements}

I gratefully acknowledge support by the NSF grants \# DMS-0503864, DMS-0352576, and am thankful to James F Davis, Erik K Pedersen, and Andrew Ranicki for helping me to locate the correct reference to the naturality of the assembly map.

\section{References}

[1] MT Anderson, Dehn filling and Einstein metrics in higher dimensions arXiv: math.DG/0303260 
[2] C S Aravinda, F T Farrell, Rank 1 aspherical manifolds which do not support any nonpositively curved metric, Comm. Anal. Geom. 2 (1994) 65-78 MR1312678

[3] I Belegradek, Aspherical manifolds with relatively hyperbolic fundamental groups arXiv: math.GR/0509490

[4] R Benedetti, C Petronio, Lectures on hyperbolic geometry, Universitext, Springer, Berlin (1992) MR1219310

[5] M R Bridson, A Haefliger, Metric spaces of non-positive curvature, Grundlehren series 319, Springer, Berlin (1999) MR1744486

[6] R M Charney, M W Davis, Strict hyperbolization, Topology 34 (1995) 329-350 MR1318879

[7] D J Collins, C F Miller, III, The word problem in groups of cohomological dimension 2, from: "Groups St. Andrews 1997 in Bath, I", London Math. Soc. Lecture Note Ser. 260, Cambridge Univ. Press (1999) 211-218 MR1676618

[8] F Dahmani, Accidental parabolics and relatively hyperbolic groups arXiv: math.GR/0210421

[9] F Dahmani, Combination of convergence groups, Geom. Topol. 7 (2003) 933-963 MR2026551

[10] J F Davis, Manifold aspects of the Novikov conjecture, from: "Surveys on surgery theory Vol 1", Ann. of Math. Stud. 145, Princeton Univ. Press (2000) 195-224

[11] M W Davis, Groups generated by reflections and aspherical manifolds not covered by Euclidean space, Ann. of Math. (2) 117 (1983) 293-324 MR690848

[12] M W Davis, Some aspherical manifolds, Duke Math. J. 55 (1987) 105-139 MR883666

[13] M W Davis, Exotic aspherical manifolds, from: "Topology of high-dimensional manifolds, No. 1, 2 (Trieste, 2001)", ICTP Lect. Notes 9, Abdus Salam Int. Cent. Theoret. Phys., Trieste (2002) 371-404 MR1937019

[14] M W Davis, T Januszkiewicz, Hyperbolization of polyhedra, J. Differential Geom. 34 (1991) 347-388 MR1131435

[15] M W Davis, T Januszkiewicz, S Weinberger, Relative hyperbolization and aspherical bordisms: an addendum to "Hyperbolization of polyhedra" by Davis and Januszkiewicz, J. Differential Geom. 58 (2001) 535-541

[16] A N Dranišnikov, D Repovšs, Embeddings up to homotopy type in Euclidean space, Bull. Austral. Math. Soc. 47 (1993) 145-148 MR1201430

[17] C Drutu, M Sapir, Tree-graded spaces and asymptotic cones of groups arXiv: math.GT/0405030

[18] M Gromov, Volume and bounded cohomology, Inst. Hautes Études Sci. Publ. Math. (1982) 5-99 (1983) MR686042 
[19] M Gromov, Hyperbolic groups, from: "Essays in group theory", Math. Sci. Res. Inst. Publ. 8, Springer, New York (1987) 75-263 MR919829

[20] M Gromov, Asymptotic invariants of infinite groups, from: "Geometric group theory, Vol. 2 (Sussex, 1991)", London Math. Soc. Lecture Note Ser. 182, Cambridge Univ. Press (1993) 1-295 MR1253544

[21] D Groves, Limit groups for relatively hyperbolic groups, I: The basic tools arXiv: math.GR/0412492

[22] I Hambleton, E K Pedersen, Identifying assembly maps in $K$ - and L-theory, Math. Ann. 328 (2004) 27-57 MR2030369

[23] J-C Hausmann, Algebraic $K$-theory and flat manifolds, from: "Algebraic topology, Aarhus 1978 (Proc. Sympos., Univ. Aarhus, Aarhus, 1978)", Lecture Notes in Math. 763, Springer, Berlin (1979) 212-234 MR561224

[24] M Hoster, D Kotschick, On the simplicial volumes of fiber bundles, Proc. Amer. Math. Soc. 129 (2001) 1229-1232 MR1709754

[25] M Kapovich, B Leeb, Actions of discrete groups on nonpositively curved spaces, Math. Ann. 306 (1996) 341-352 MR1411351

[26] T Kuessner, Multicomplexes, bounded cohomology and additivity of simplicial volume arXiv:math.AT/0109057

[27] J-L Lafont, B Schmidt, Simplicial volume of closed locally symmetric spaces of non-compact type arXiv:math.DG/0504338

[28] G Mess, Examples of Poincaré duality groups, Proc. Amer. Math. Soc. 110 (1990) 1145-1146 MR1019274

[29] C F Miller, III, Decision problems for groups—survey and reflections, from: "Algorithms and classification in combinatorial group theory (Berkeley, CA, 1989)", Math. Sci. Res. Inst. Publ. 23, Springer, New York (1992) 1-59 MR1230627

[30] I Mineyev, Straightening and bounded cohomology of hyperbolic groups, Geom. Funct. Anal. 11 (2001) 807-839 MR1866802

[31] L Mosher, M Sageev, Nonmanifold hyperbolic groups of high cohomological dimension, preprint (1997) Available at http://newark.rutgers.edu/ mosher/

[32] B Okun, Exotic smooth structures on nonpositively curved symmetric spaces, Algebr. Geom. Topol. 2 (2002) 381-389 MR1917058

[33] P Ontaneda, The double of a hyperbolic manifold and non-positively curved exotic PL structures, Trans. Amer. Math. Soc. 355 (2003) 935-965 MR1938740

[34] DV Osin, Relatively hyperbolic groups: Intrinsic geometry, algebraic properties, and algorithmic problems arXiv:math.GR/0404040

[35] D V Osin, Small cancellations over relatively hyperbolic groups and embedding theorems arXiv:math.GR/0411039 
[36] J G Ratcliffe, S T Tschantz, Some examples of aspherical 4-manifolds that are homology 4-spheres, Topology 44 (2005) 341-350 MR2114711

[37] V Schroeder, A cusp closing theorem, Proc. Amer. Math. Soc. 106 (1989) 797-802 MR957267

[38] J Smillie, Flat manifolds with non-zero Euler characteristics, Comment. Math. Helv. 52 (1977) 453-455 MR0461521

[39] T Soma, The Gromov invariant of links, Invent. Math. 64 (1981) 445-454 MR632984

[40] J R Stallings, Embedding homotopy types into manifolds, preprint (1965) Available at http://math. berkeley.edu/ stall/

[41] T Yamaguchi, Simplicial volumes of Alexandrov spaces, Kyushu J. Math. 51 (1997) 273-296 MR1470157

[42] A Yaman, A topological characterisation of relatively hyperbolic groups, J. Reine Angew. Math. 566 (2004) 41-89 MR2039323

School of Mathematics, Georgia Institute of Technology

Atlanta GA 30332-0160

ib@math.gatech.edu

Received: 19 October 2005 